تقتية إضافة وحدات التوليد الصغيرة باستخدام الخوارزمية الجينية لتقليل قيمة الفقد

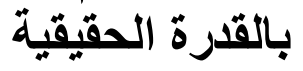

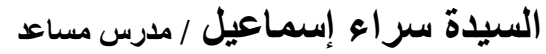

قسم الهندسة الكهربائية / جامعة الموصل

\begin{abstract}
الملخص

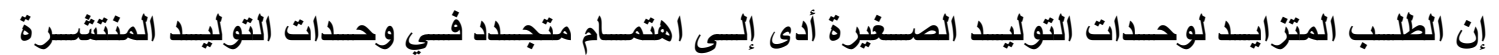

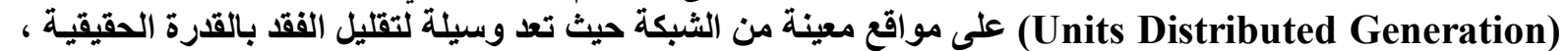

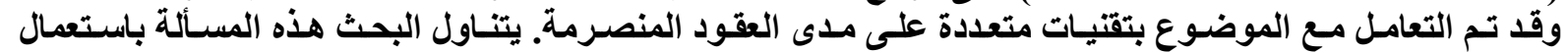

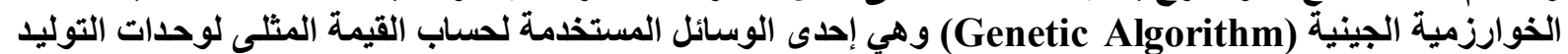

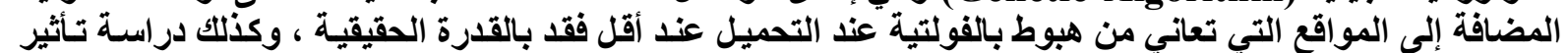

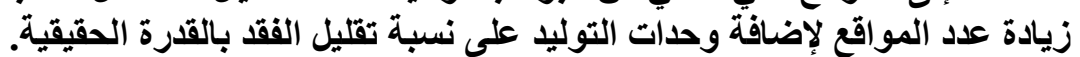

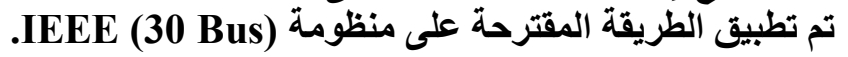

\title{
Genetic Algorithm Based Small Distributed Generation Units Addition Technique To Reduce Real Power Losses
}

\author{
Saraa Esmaeel Khaleel / Assist. Lecturer \\ Electrical Engineering Department \\ University of Mosul
}

\begin{abstract}
The increasing demand on installing small generating units had resulted in focusing on advanced concern of distributed generation (DG) units located at specific points of the electrical distribution grid as a mean of reduction in real power losses. This issue had been dealt with many techniques over the last decades.

This research paper is taking Genetic Algorithm technique into consideration as one of the most efficient tools in calculating the optimum value of these DG units added to the grid locations suffering from loading effect based voltage drop at the minimum real power loss. This research is also concerning the impact of increasing number of locations of DG units added to the grid the percentage of reduction in real power loss.
\end{abstract}

Keywords: Small Generator Units, Genetic Algorithm, Optimal (DG) Units, Add to Grid Real Power Losses 


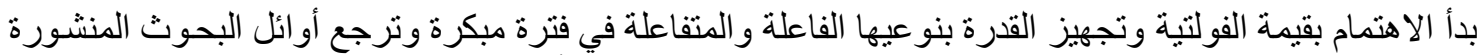

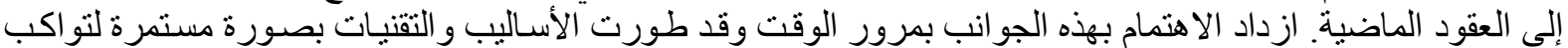

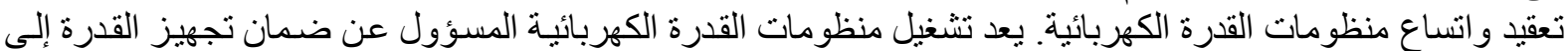

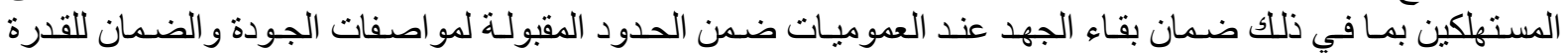

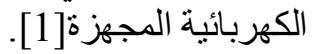

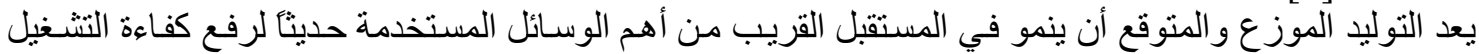

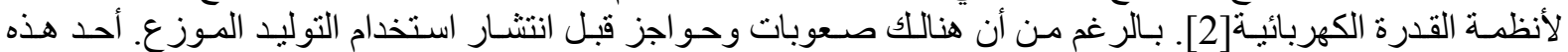

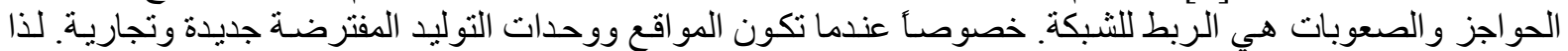

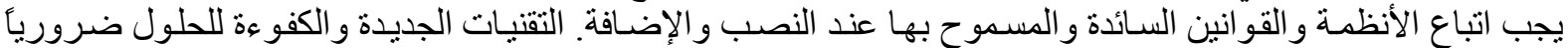
لجعل التوليد الموزع القنتصادياً ومتوفرًا.

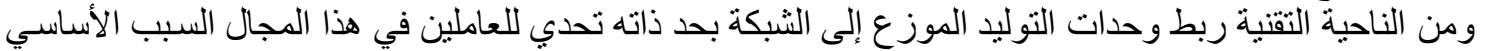

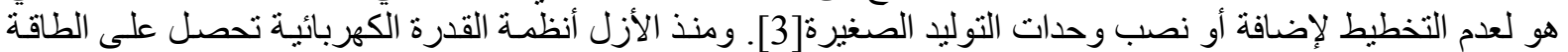

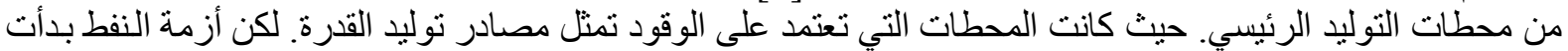

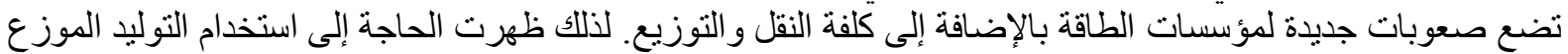

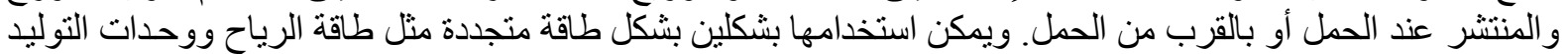

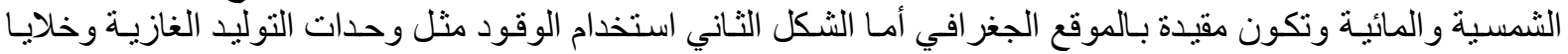
الوقود. و هذا التوليد الموزع الصغير يبين نتائج عالية في تقليل مقدار الفقد بالقدرة الحقيقية. بالإضـافة إلى تقليل الإجهاد

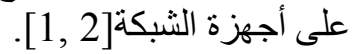
وقد استخدمت الخوارزمية الجينية (Genetic Algorithm) في إيجاد التشـيل الأمثل لتعويض القدرة الفاعلـة في

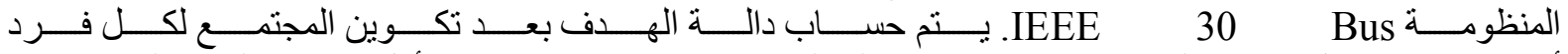
أي حساب كفاءة كل فرد في المجتمع وقد تم اعتبـار دالة الهدف (Fitness Function) أقل خسارة بالقدرة الحقيقيـة. ينت البحث عن القيمة المنلى لحقن وحدات التوليد المنتشرة التي تجعل دالة الهدف أقل ما يمكن [4].

Fitness Function $=\mathrm{P}_{\text {Loss }}$

$\mathrm{P}_{\text {Loss }}=\sum_{\mathrm{i}=1}^{\mathrm{N}} \sum_{\mathrm{j}=1}^{\mathrm{N}} \mathrm{G}_{\mathrm{ij}}\left[\left|\mathrm{V}_{\mathrm{i}}\right|^{2}+\left|\mathrm{V}_{\mathrm{j}}\right|^{2}-2\left|\mathrm{~V}_{\mathrm{i}}\right|\left|\mathrm{V}_{\mathrm{j}}\right| \operatorname{Cos}\left(\delta_{\mathrm{i}}-\delta_{\mathrm{j}}\right)\right]$

: P Pass

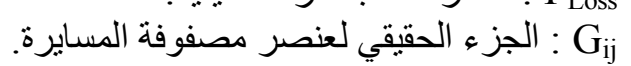

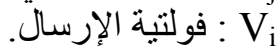

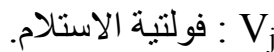

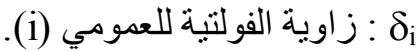

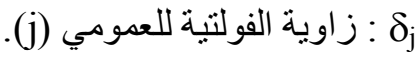

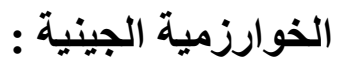

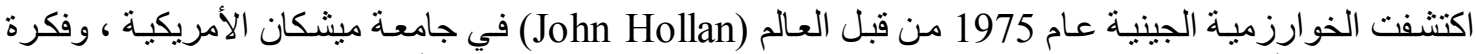

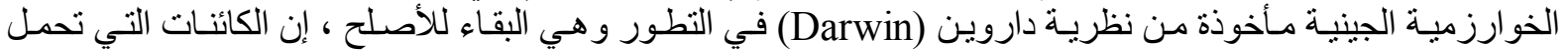

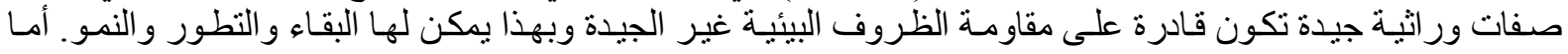

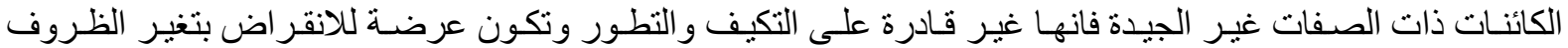

المناخية[5].

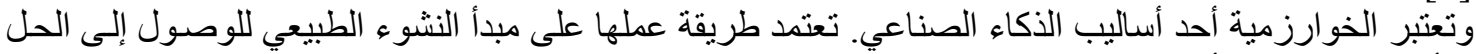

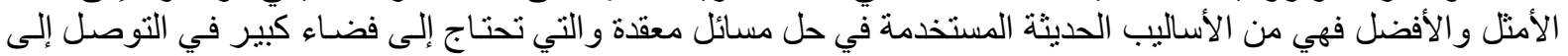

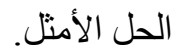

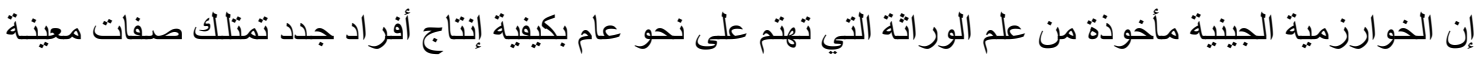

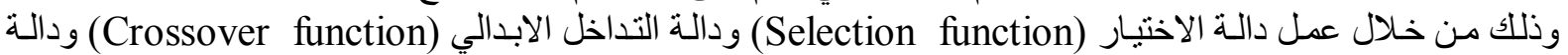
الطفرة (Mutation function) التي تحدث على المجموعات المورثة بهدف تكوين أفر اد جدد] (6]. 


\section{أهمية وعمل ( الخوارزمية الجينية ) :}

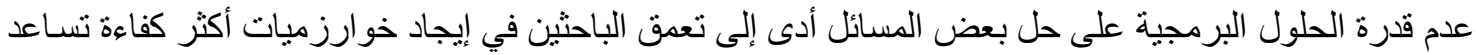

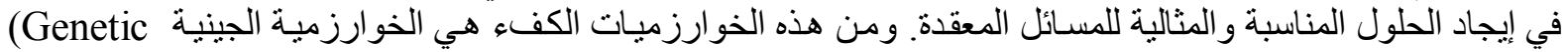
.Algorithm)

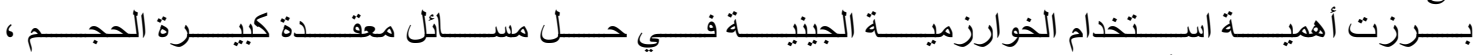

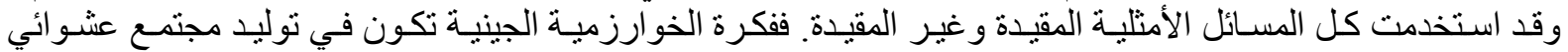

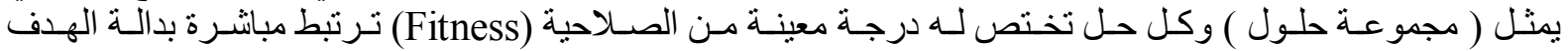

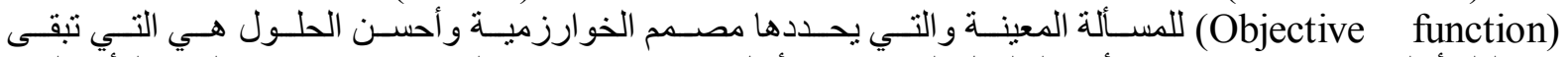

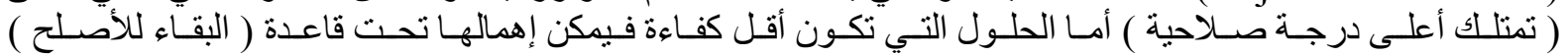

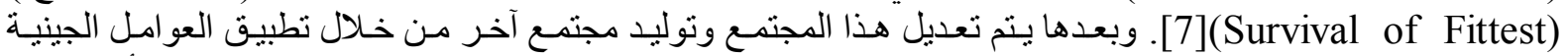

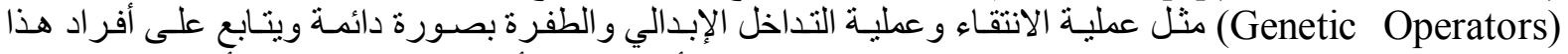

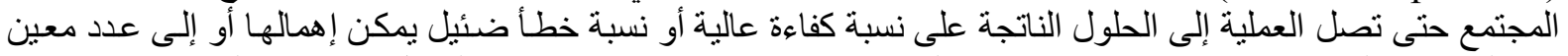

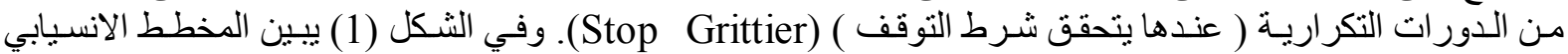
للخطو ات العامة للخوارزمية الجينية.

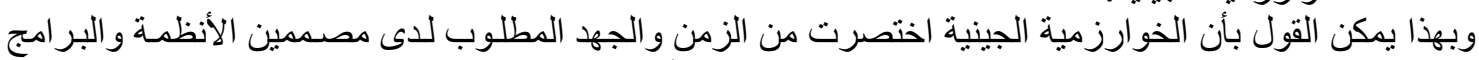

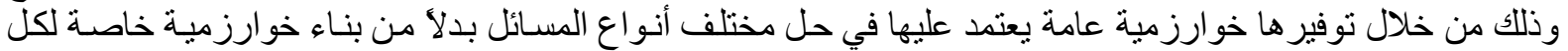

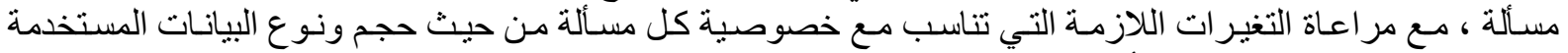

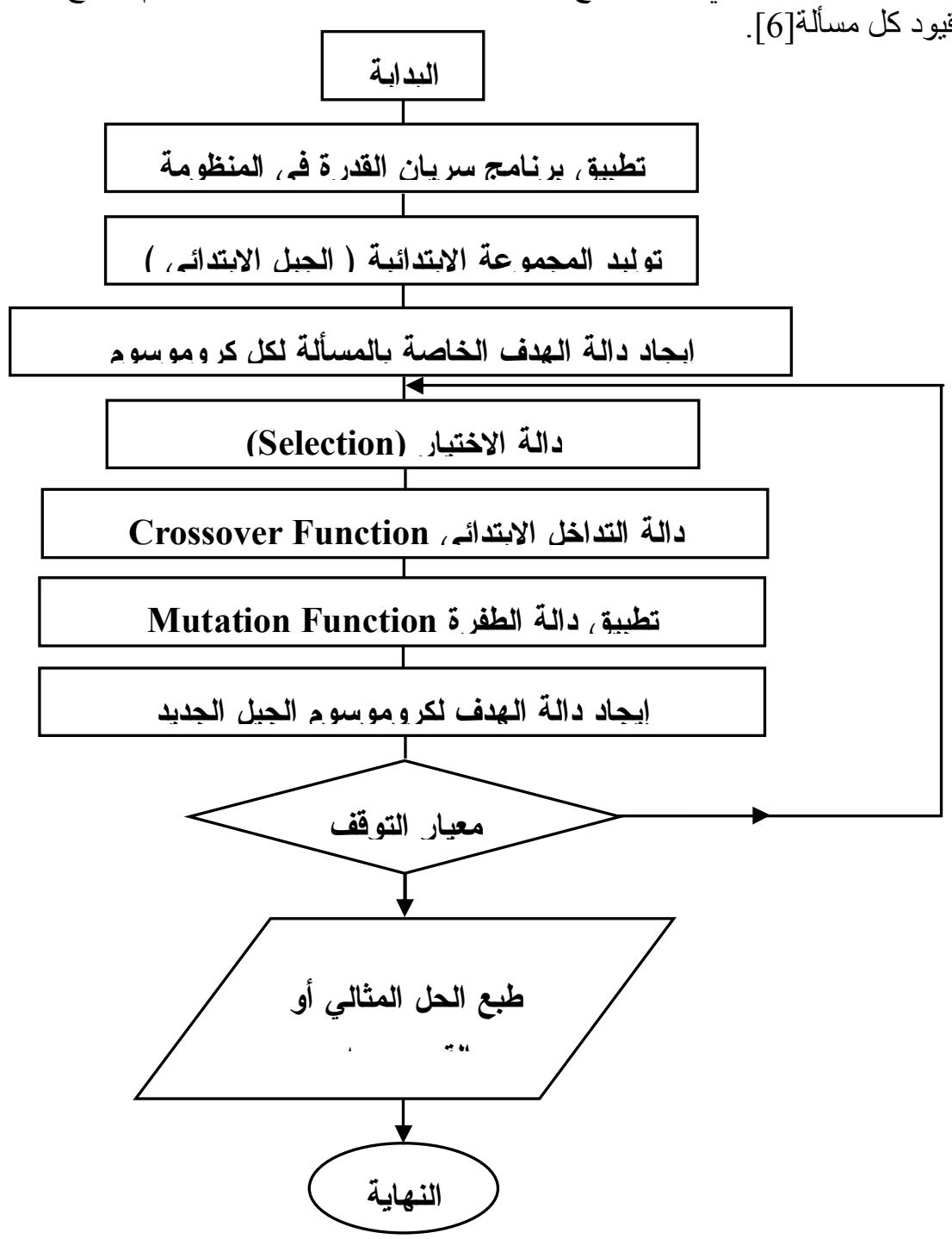

الثكل (1) : المخطط الانسيابِ للخطوات العامة للخوارزمية الجينية 


\section{النتائج ومناقشتها :}

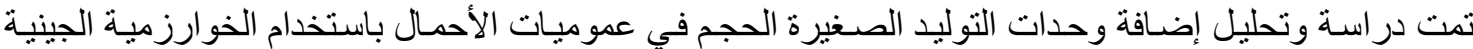

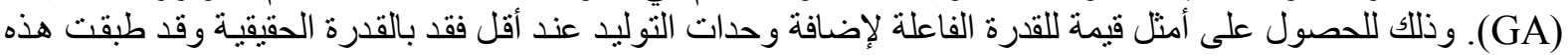
الخوارزمية على المنظومة (IEAE 30 bus) في حالة التحميل فقد كانت قيمة الفقد بالقدرة الحقيقيـة بسـاوي .MW) وقد استخدمت الخوارزميـة الجينيـة لإضـافة وحدات التوليد صـغيرة الحجم على المو اقـع التي تعـاني من هبوط بالفولتية ومقارنتها مع طريقة المشتقة الضمنية لنفس الثبكة] [4].

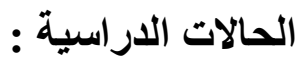

1- إضافة وحدات التوليد صغيرة الحجم لموقع واحد.

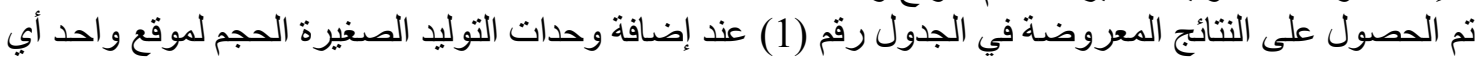

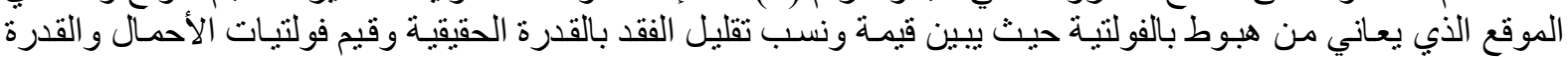
المتفاعلة لعمو ميات التوليد عند الإضافة المنلى لوحدات التوليد.

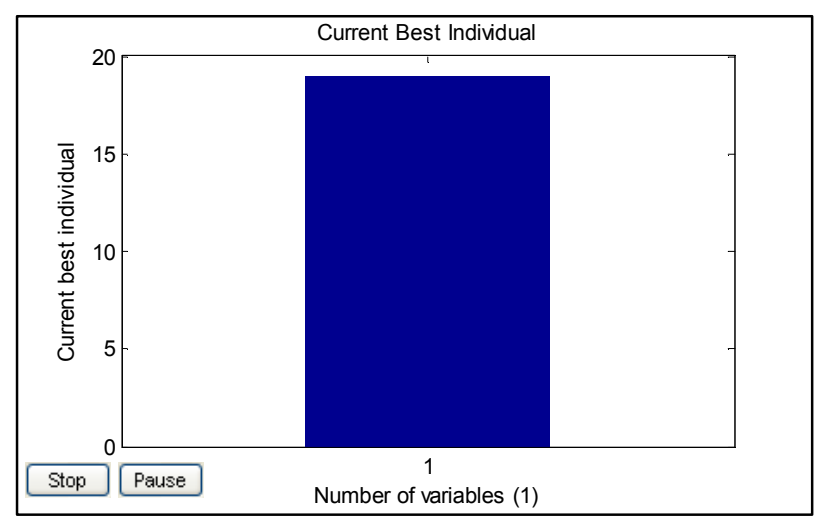

الشكل (3) : بيين علاقة عدد وحدات التوليا المضافة

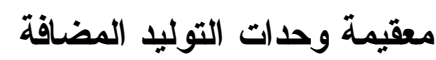

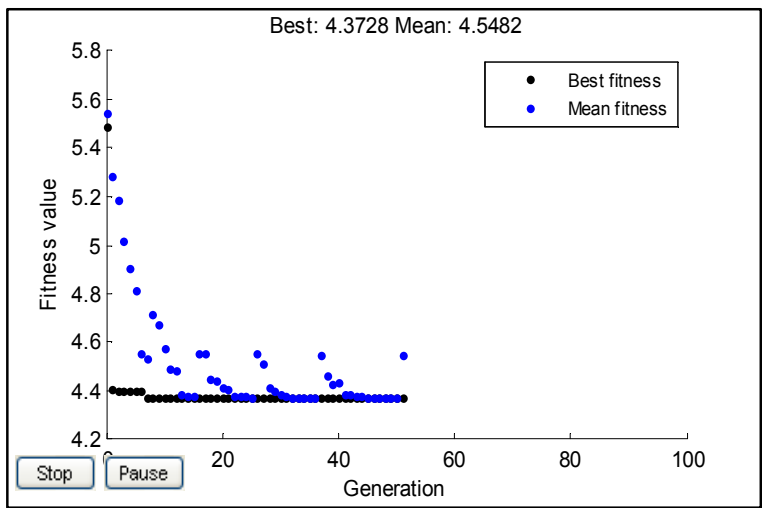

الشكل (2) : يبين علاقة عدد الأجيال المتولدة مع دالة الهدف ( الفقد بالقدرة الحقيقية عند الإضافة لموقع واحد )

2- إضافة وحدات التوليد صغيرة الحجم لثلاث مو اقع تعاني من هبوط بالفولتية.

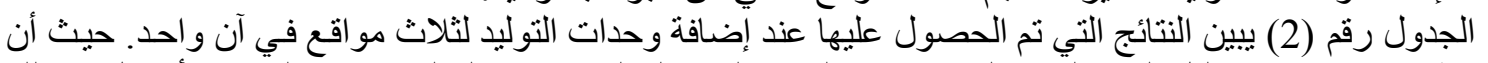

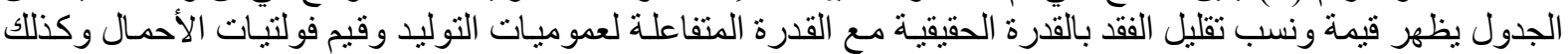
القيمة المنلى المضافة لوحدات التوليد.

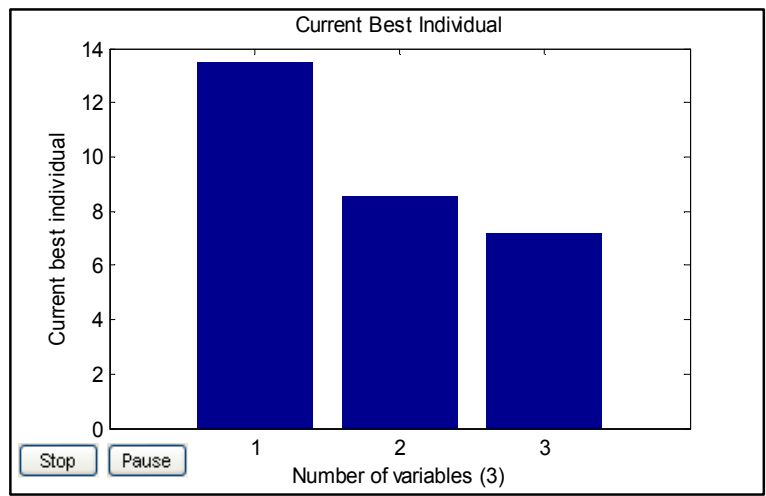

الشكل (5) : بيينة علاقة عدد وحدات التوليد

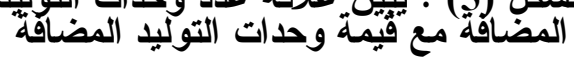

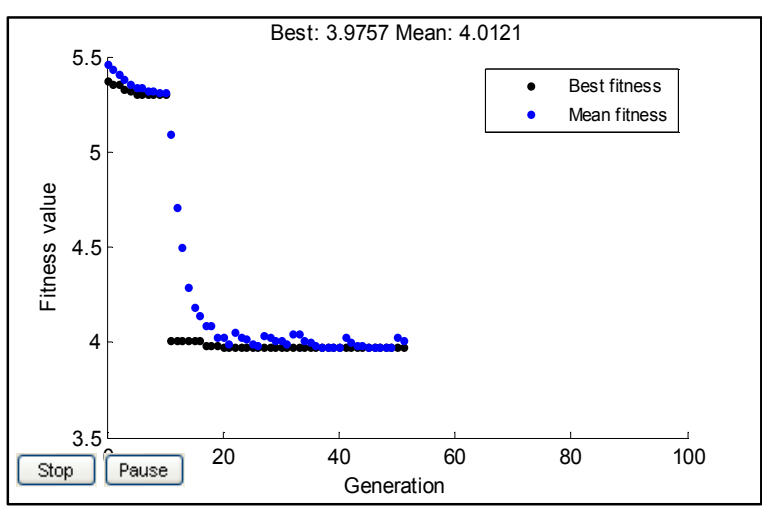

الشيكل (4) : يبيني علاقه عدد الإيبيل المتولدة ميع

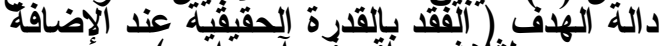
لثثلاث موأقع في آن و"اخد ) 


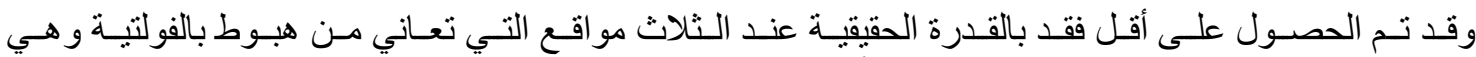

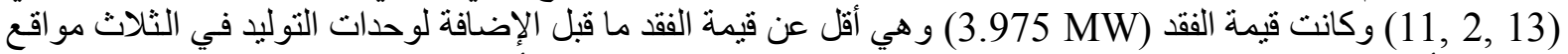

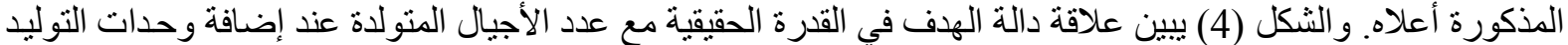
في الثناث مو اقع (11, 2, 13) كما وأن الثكل (5) ييين قيمة وحدات التولئ ليد المضافة.

3- إضافة وحدات التوليد صغيرة الحجم لأربع مو اقع تعاني من هبوط بالفولتية.

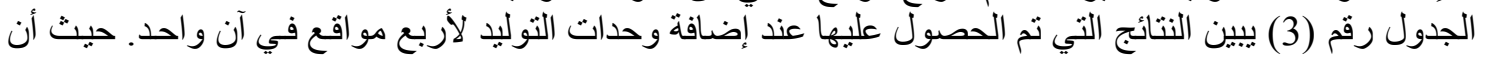

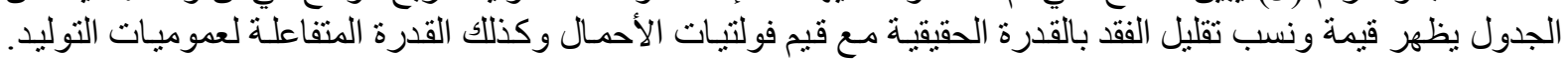
وكذلك القيمة المثلى المضافة لوحدات التولئل التوليد.

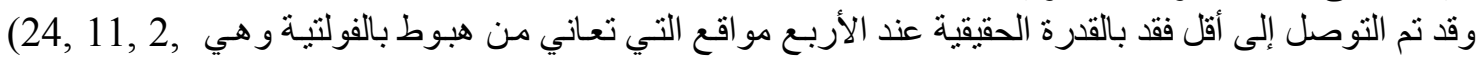
(13 وكانت قيمة الفقد (3.061 MW) ونسبة تقليل الفقد (45.3\%).

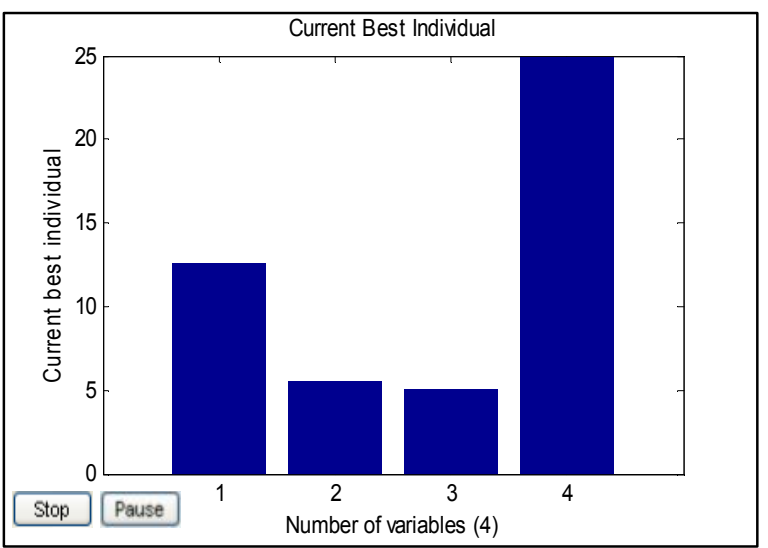

الشكل (7) : يبين علاقة عدد وحدات التوليا

المضافة مع قيمة وحدات التوليد المضافة

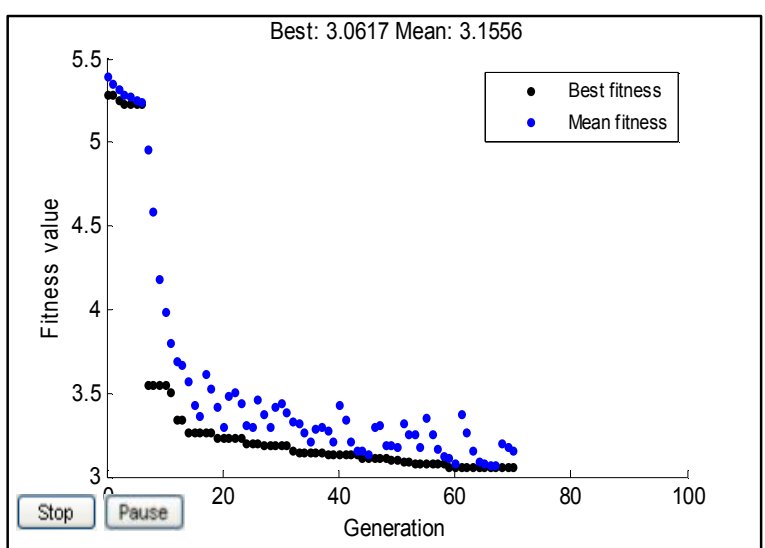

الشكل (6) : يبين علاقة عدد الأجيال المتولدة مع دالة الههف ( الفقد بالقدرة الحقيقية عند الإضافة لأربع مواقع في آن واحد )

4- إضافة وحدات التوليد صغيرة الحجم لست مواقع في آن واحد.

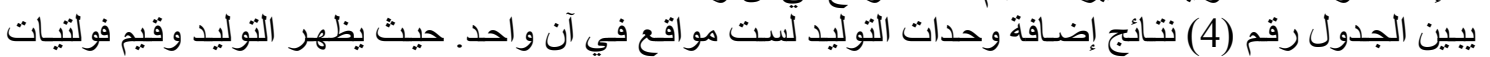

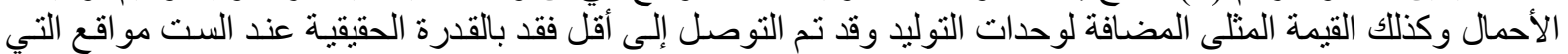
تعاني من هبوط بالفولتيـة وهي (2.342 MW) و ونسبة تقليل الفقد

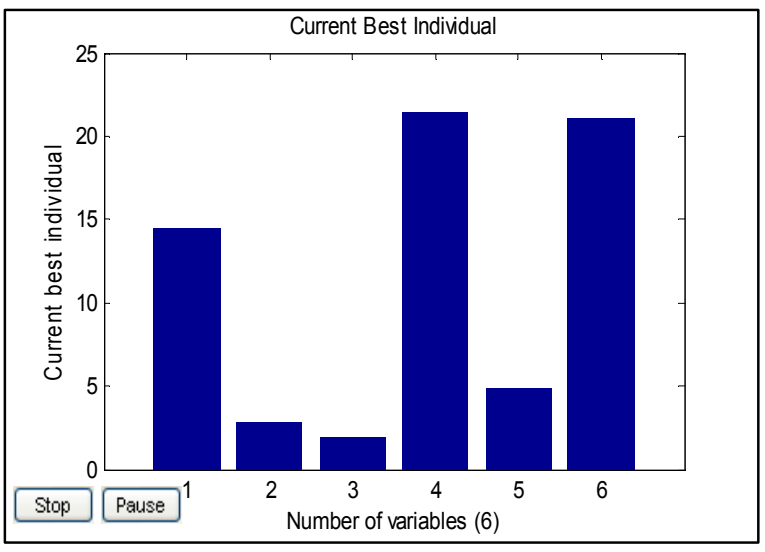

الشكل (9) : يبين علاقة عدد وحدات التوليد

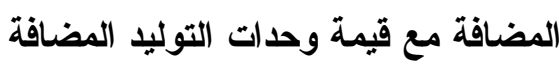

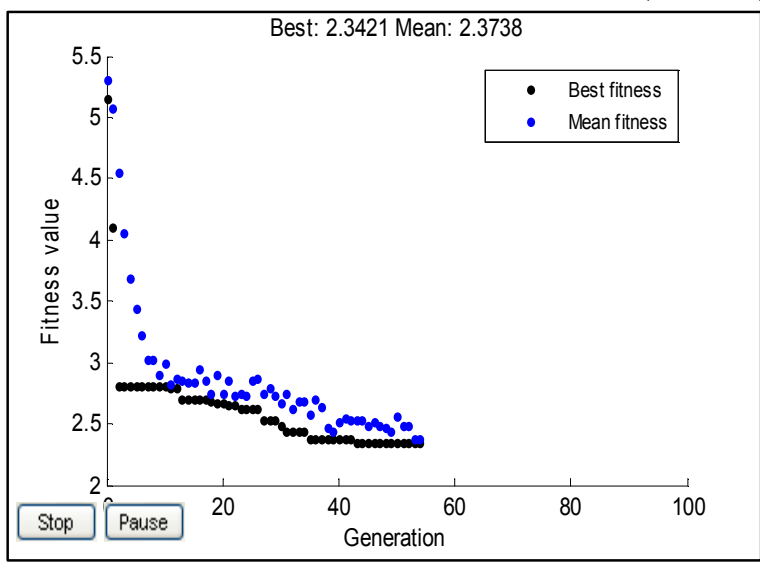

ألثكل(8) : يبين علاقة عدد الأجيال المتولدة مع دالة

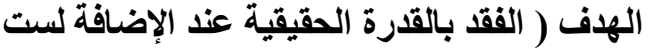
مواقع في آن واحد ) 

1- إن الفقد بالقدرة الحقيقية يكون أقل كلما يزداد عدد وحدات التوليد المضافة على العموميات التي تعاني من هبوط بالفولتية. 2- أعطت طريقة الخوارزمية الجينية سهولة في الحل وكذلك تم تقليل الفقد بشكل أكبر من الطرق التقليديـة السـابقة

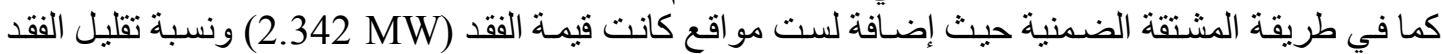
(58.2\%) إذا ما قورنت بطريقة المشتقة الضمنية لنفس مو اقع حيث كانت قيمـة الفقد (2.449 MW) ونسبة تقليل الفقد (56.29\%).

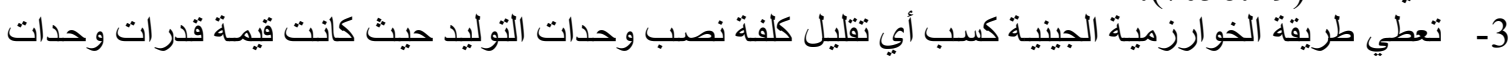
التوليد المضافة لست مو اقع (67.7 MW) بينما في طريقة المشتقة الضمنية لنفس المو اقع هي (77.5 MW).

الجدول (1) : يبين قيم ونسب تقليل الفقد بالقدرة الحقيقية وقيم فولتيات الأحمال والقدرة المتفاعلة لعموميات التوليد عند

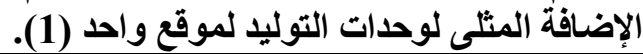

\begin{tabular}{|c|c|c|}
\hline \multirow{2}{*}{ متغيرات المسألة } & الإضافة المثلى لوحدات التوليا & الحالة الاعتيادية \\
\hline & \multicolumn{2}{|c|}{ 1- القرة المتفاعلة لعموميات التوليا (MVAR) } \\
\hline QG1 & 2.3131 & -2.09 \\
\hline QG26 & 56.5599 & 50.366 \\
\hline QG27 & 20.4519 & 21.3734 \\
\hline QG28 & 29.3806 & 34.244 \\
\hline QG29 & 20.5663 & 21.0365 \\
\hline QG30 & 27.0835 & 27.2952 \\
\hline \multicolumn{3}{|c|}{ 2- فولتيات عموميات الأحمال (P.U) } \\
\hline VL2 & 0.9565 & 0.9452 \\
\hline VL3 & 1.0233 & 1.0219 \\
\hline VL4 & 1.0177 & 1.0159 \\
\hline VL5 & 0.9892 & 0.9741 \\
\hline VL6 & 1.0116 & 1.0096 \\
\hline VL7 & 1.0021 & 1.008 \\
\hline VL8 & 1.0083 & 1.0023 \\
\hline VL9 & 1.0100 & 1.0091 \\
\hline VL10 & 0.9891 & 0.9885 \\
\hline VL11 & 0.9865 & 0.9531 \\
\hline VL12 & 1.0142 & 1.014 \\
\hline VL13 & 0.9936 & 0.941 \\
\hline VL14 & 0.9969 & 0.9964 \\
\hline VL15 & 0.9908 & 0.9897 \\
\hline VL16 & 0.9962 & 0.9957 \\
\hline VL17 & 0.9857 & 0.985 \\
\hline VL18 & 0.9774 & 0.9765 \\
\hline VL19 & 0.9728 & 0.9719 \\
\hline VL20 & 0.9761 & 0.9752 \\
\hline VL21 & 0.9762 & 0.9751 \\
\hline VL22 & 0.9769 & 0.9756 \\
\hline VL23 & 0.9765 & 0.9714 \\
\hline VL24 & 0.9663 & 0.9622 \\
\hline VL25 & 0.9750 & 0.9639 \\
\hline قيمة الفقد (MW) & $4.372 \mathrm{MW}$ & $5.6053 \mathrm{MW}$ \\
\hline نسبة تقليل (\%) & $22 \%$ & \\
\hline قيمة وحدات التوليد الكلية (PGT) & $18.999 \mathrm{MW}$ & \\
\hline
\end{tabular}


اسماعيل: تقنية إضافة وحدات التوليد الصغيرة باستخدام الخوارزمية الجينية لتقليل قيمة الفقد بالقدرة الحقيقية

الجدول (2) : يبين قيم ونسب تقليل الفقد بالقدرة الحقيقية وقيم فولتيات الأحمال والقدرة المتفاعلة لعموميات التوليد علديات الإضافة المثلى لوحدات التوليد لثثلاث مواقع (13, 1 الإنة

\begin{tabular}{|c|c|c|}
\hline متغيرات المسألة & الإضافة المثلى لوحدات التوليا & الحالة الاعتيادية \\
\hline \multicolumn{3}{|c|}{ 1- القدرة المتفاعلة لعموميات التوليا (MVAR) } \\
\hline QG1 & 5.8209 & -2.09 \\
\hline QG26 & 44.1986 & 50.366 \\
\hline QG27 & 19.9533 & 21.3734 \\
\hline QG28 & 26.6323 & 34.244 \\
\hline QG29 & 20.3449 & 21.0365 \\
\hline QG30 & 27.1010 & 27.2952 \\
\hline \multicolumn{3}{|c|}{ 2- فولتيات عموميات الأحمال (P.U) } \\
\hline VL2 & 0.9904 & 0.9452 \\
\hline VL3 & 1.0242 & 1.0219 \\
\hline VL4 & 1.0188 & 1.0159 \\
\hline VL5 & 0.9960 & 0.9741 \\
\hline VL6 & 1.0129 & 1.0096 \\
\hline VL7 & 1.0029 & 1.008 \\
\hline VL8 & 1.0116 & 1.0023 \\
\hline VL9 & 1.0105 & 1.0091 \\
\hline VL10 & 0.9893 & 0.9885 \\
\hline VL11 & 1.0057 & 0.9531 \\
\hline VL12 & 1.0142 & 1.014 \\
\hline VL13 & 1.0008 & 0.941 \\
\hline VL14 & 0.9970 & 0.9964 \\
\hline VL15 & 0.9915 & 0.9897 \\
\hline VL16 & 0.9962 & 0.9957 \\
\hline VL17 & 0.9858 & 0.985 \\
\hline VL18 & 0.9779 & 0.9765 \\
\hline VL19 & 0.9732 & 0.9719 \\
\hline VL20 & 0.9764 & 0.9752 \\
\hline VL21 & 0.9768 & 0.9751 \\
\hline VL22 & 0.9776 & 0.9756 \\
\hline VL23 & 0.9785 & 0.9714 \\
\hline VL24 & 0.9679 & 0.9622 \\
\hline VL25 & 0.9865 & 0.9639 \\
\hline قيمة الفقد (MW) & $3.975 \mathrm{MW}$ & $5.6053 \mathrm{MW}$ \\
\hline نسبة تقليل (\%) & $29.08 \%$ & \\
\hline قيمة وحدات التوليد الكلية (PGT) & $29.33 \mathrm{MW}$ & \\
\hline
\end{tabular}




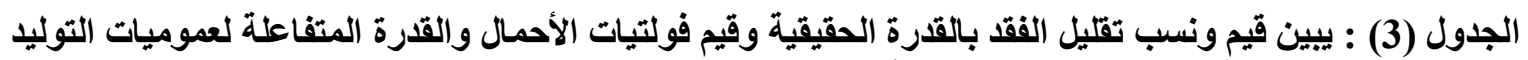
الإضافة المثلى لوحدات التوليد لأربع مواقع (13, 2, 11, 24).

\begin{tabular}{|c|c|c|}
\hline متغيرات المسألة & الإضافة المثلى لوحدات التوليا & الحالة الاعتيادية \\
\hline \multicolumn{3}{|c|}{ 1- القدرة المتفاعلة لعموميات التوليد (MVAR) } \\
\hline QG1 & 5.6021 & -2.09 \\
\hline QG26 & 44.1313 & 50.366 \\
\hline QG27 & 19.9408 & 21.3734 \\
\hline QG28 & 26.4285 & 34.244 \\
\hline QG29 & 19.9943 & 21.0365 \\
\hline QG30 & 26.6399 & 27.2952 \\
\hline \multicolumn{3}{|c|}{ 2- فولتيات عموميات الأحمال (P.U) } \\
\hline VL2 & 0.9821 & 0.9452 \\
\hline VL3 & 1.0243 & 1.0219 \\
\hline VL4 & 1.0190 & 1.0159 \\
\hline VL5 & 0.9937 & 0.9741 \\
\hline VL6 & 1.0129 & 1.0096 \\
\hline VL7 & 1.0024 & 1.008 \\
\hline VL8 & 1.0106 & 1.0023 \\
\hline VL9 & 1.0112 & 1.0091 \\
\hline VL10 & 0.9406 & 0.9885 \\
\hline VL11 & 0.9937 & 0.9531 \\
\hline VL12 & 1.0143 & 1.014 \\
\hline VL13 & 0.9916 & 0.941 \\
\hline VL14 & 0.9980 & 0.9964 \\
\hline VL15 & 0.9932 & 0.9897 \\
\hline VL16 & 0.9972 & 0.9957 \\
\hline VL17 & 0.9870 & 0.985 \\
\hline VL18 & 0.9795 & 0.9765 \\
\hline VL19 & 0.9747 & 0.9719 \\
\hline VL20 & 0.9779 & 0.9752 \\
\hline VL21 & 0.9790 & 0.9751 \\
\hline VL22 & 0.9801 & 0.9756 \\
\hline VL23 & 0.9823 & 0.9714 \\
\hline VL24 & 0.9766 & 0.9622 \\
\hline VL25 & 0.9857 & 0.9639 \\
\hline قيمة الفقد (MW) & $3.061 \mathrm{MW}$ & $5.6053 \mathrm{MW}$ \\
\hline نسبة تقليل (\%) & $45.3 \%$ & \\
\hline قيمة وحدات التوليد الكلية (PGT) & $47 \mathrm{MW}$ & \\
\hline
\end{tabular}


اسماعيل: تقنية إضافة وحدات التوليد الصغيرة باستخدام الخوارزمية الجينية لتقليل قيمة الفقد بالقدرة الحقيقية

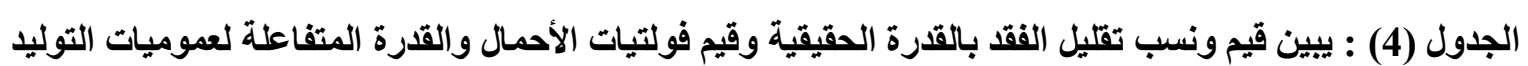

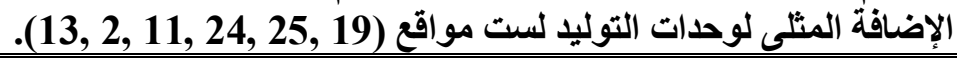

\begin{tabular}{|c|c|c|}
\hline \multirow{2}{*}{ متغيرات المسألة } & الإضافة المثلى لوحدات التوليد & الحالة الاعتيادية \\
\hline & \multicolumn{2}{|c|}{ 1- القدرة المتفاعلة لعموميات التوليا (MVAR) } \\
\hline QG1 & 14.9622 & -2.09 \\
\hline QG26 & 38.8618 & 50.366 \\
\hline QG27 & 18.9749 & 21.3734 \\
\hline QG28 & 20.1138 & 34.244 \\
\hline QG29 & 18.6190 & 21.0365 \\
\hline QG30 & 25.6286 & 27.2952 \\
\hline \multicolumn{3}{|c|}{ 2- فولتيات عموميات الأحمال (P.U) } \\
\hline VL2 & 0.9956 & 0.9452 \\
\hline VL3 & 1.0267 & 1.0219 \\
\hline VL4 & 1.0221 & 1.0159 \\
\hline VL5 & 0.9959 & 0.9741 \\
\hline VL6 & 1.0155 & 1.0096 \\
\hline VL7 & 1.0045 & 1.008 \\
\hline VL8 & 1.0140 & 1.0023 \\
\hline VL9 & 1.0139 & 1.0091 \\
\hline VL10 & 0.9945 & 0.9885 \\
\hline VL11 & 0.9919 & 0.9531 \\
\hline VL12 & 1.0162 & 1.014 \\
\hline VL13 & 0.9936 & 0.941 \\
\hline VL14 & 1.0041 & 0.9964 \\
\hline VL15 & 1.0009 & 0.9897 \\
\hline VL16 & 0.9997 & 0.9957 \\
\hline VL17 & 0.9906 & 0.985 \\
\hline VL18 & 0.9961 & 0.9765 \\
\hline VL19 & 0.9965 & 0.9719 \\
\hline VL20 & 0.9950 & 0.9752 \\
\hline VL21 & 0.9860 & 0.9751 \\
\hline VL22 & 0.9927 & 0.9756 \\
\hline VL23 & 0.9906 & 0.9714 \\
\hline VL24 & 0.9959 & 0.9622 \\
\hline VL25 & 1.0450 & 0.9639 \\
\hline قيمة الفقد (MW) & $2.342 \mathrm{MW}$ & $5.6053 \mathrm{MW}$ \\
\hline نسبة تقليل (\%) & $58.2 \%$ & \\
\hline قيمة وحدات التوليد الكلية (PGT) & $67.7 \mathrm{MW}$ & \\
\hline
\end{tabular}




\section{References:}

1. B. F. Ahmed, "Optimal Reactive Power Value in Mosul Ring Bus-bar Using Genetic Algorithm". M. Sc. Thesis, University of Mosul, 2009.

2. T. Griffin, K. Tomsovic, D. Secrest and A. Law "Placement of Dispersed Generation System for Reduced Losses". Proceeding of the $33^{\text {rd }}$ Hawaii International Conference on System Science-2000.

3. Kimmo Kauhaniemi ... etc. "Distribution Generation-NeE Technical Solution Required the Distribution System", Proceeding of ESPOO Conference August, 23-24, 2004, Finland.

4. S. E. Khaleel "Reduction of Generation-Cost and Losses by Optimal Control of Active and Reactive Power". M. Sc. Thesis, University of Mosul, 2004.

5. Obitko, M. (1998). "Genetic Algorithms". http://www.cs.felk.cvut.cz/ xobitko/gar.

6. De Gold Beery. "Genetic Algorithms in Search Optimization, and Learning". Reading, MA: Addition Wesley, 1998.

7. Genetic Algorithm and Direct Search Toolbox2 User's Guide. "The Math Works". Inc. 2007.

$$
\text { تم اجراء البحث في كلية ألهندسة = جامعة ألموصل }
$$

